\title{
¿Afecta la fase de juego al diseño de las tareas de un equipo de baloncesto de formación?
}

\section{Does the game phase affect the design of tasks for a formative basketball team?}

\author{
David Mancha Triguero ${ }^{1,4}$, Juan Manuel García Ceberino ${ }^{2,4}$, Antonio Antúnez Medina ${ }^{2,4}$ y Javier García Rubio ${ }^{1,3,4}$ \\ 1 Facultad Ciencias del Deporte. Universidad de Extremadura. Cáceres (España). \\ 2 Facultad de Educación. Universidad de Extremadura. Badajoz (España). \\ 3 Facultad de Educación. Universidad Autónoma de Chile. Santiago de Chile (Chile). \\ 4 Grupo de Optimización del Entrenamiento y Rendimiento Deportivo. (GOERD).
}

\begin{abstract}
Resumen: Durante la temporada, el papel principal del entrenador es el de diseńar y plantear las tareas que ayuden a conseguir los objetivos planteados de manera secuencial. El objetivo de este trabajo fue describir si existen diferencias entre las tareas que son diseñadas en función de la fase de juego. Para ello, se recogieron 388 tareas durante una temporada de un equipo de baloncesto. Esta comparativa se debe a que el entrenador, realiza los entrenamientos bajo enfoque comprensivo y elige un modelo ondulatorio del trabajo en el que se alternan tareas con finalidad de ataque y defensa. Finalmente, este trabajo presenta un análisis crítico y fiable sobre el diseño y el planteamiento de los entrenamientos con una visión global que recoge las diferencias encontradas en las tareas en función de la fase de juego.

Palabras clave: Entrenamiento; Tareas; Fase de Juego; Entrenador; Baloncesto.
\end{abstract}

Abstract: During the season, the main role of the coach is to design and plan the tasks that help achieve the objectives sequentially set. The aim of this study is to describe the existing differences between the tasks designed in function of the game phase. To do so, 388 tasks were collected during a season of a basketball team. This comparison is due to the fact that the coach performs the training under a comprehensive approach and chooses a wave model of work in which tasks are alternated with an aim of attack and defense. Finally, this study presents a critical and reliable analysis on the design and training plan with a global vision that shows the differences found in the tasks in function of the game phase.

Keywords: Training; Tasks; Game phase; Coach; Basketball.

\section{Introducción}

El baloncesto es un deporte de cooperación/oposición, dinámico, en el que se realizan desplazamientos rápidos y cortos acompañados en ocasiones de saltos (Narazaki, Berg, Stergiou, \& Chen, 2009). La organización de las competiciones se estructura en función de la edad de los jugadores. Cada período formativo del jugador precisa un tratamiento diferenciado de los contenidos, empleando para ello los medios de entrenamiento más adecuados para su desarrollo (Cañadas, Ibáńez, García, Parejo, \& Feu, 2010). Este proceso es el medio con el que el entrenador provoca unas mejoras educativas, comportamentales y deportivas (Arias-Estero, 2008). Todo proceso requiere de buena programación y aunar una serie de características tales como ser sistemática (completa y coherente), realizable (realista y ajustada al nivel de los jugadores), flexible, creativa (recoger aportaciones del grupo), que tenga prospectiva (anticiparse a lo que pueda ocurrir), que sea un proceso dinámico (en continua trasformación y mejora) y autocritica (reflexión sobre nuestra actuación) (Giménez \& Sáenz- López, 2000).

Dirección para correspondencia [Correspodence address]: David Mancha Triguero. Laboratorio de Optimización del Entrenamiento (Grupo GOERD). Campus Universitario, Av. De la Universidad, S/N, 10003. Cáceres, Cáceres (España). E-mail: dmanchat@alumnos.unex.es
El análisis del proceso formativo toma mayor importancia en deportes de colaboración- oposición en el que se intentan imitar las exigencias de la competición en un entorno no competitivo (Ford, Yates, \& Williams, 2010) con el fin de conocer y analizar la carga de entrenamiento (Cañadas \& Ibáñez, 2010; Cañadas, Ibáñez, García, Parejo \& Feu, 2012). Este análisis también es relevante para el entrenador ya que obtiene una información propia de como dirige y diseña el proceso de formación de su grupo pudiendo utilizarlo como Feedback (Cañadas, Gómez-Ruano, García-Rubio \& Ibáñez, 2018).

El entrenador es el máximo responsable del proceso de formación (Feu \& Ibáñez, 2001) asumiendo las funciones de planificar, diseñar y llevar a cabo el proceso de entrenamiento deseado puesto que es básico para conocer objetivamente como se realiza el entrenamiento en deportes colectivos (Ibánez, 2008). Analizar la figura del entrenador y la metodología que utiliza es un fenómeno que actualmente está en auge (Cassidy, Jones, \& Potrac, 2004). En la actualidad existen multitud de investigaciones con el entrenador como objeto de estudio, siendo el análisis de esta figura muy amplio y variado. La metodología empleada por el entrenador en el proceso puede ser muy variada, viéndose afectada por el proceso de formación que ha llevado a cabo, experiencias 
previas y capacidad de innovación (Clemente, Martins, \& Mendes, 2015).

Todo esto anteriormente desarrollado puede tener dos grandes vertientes, los entrenadores que se encuentran bajo un modelo técnico basado en la repetición de gestos técnicos $y$, por otra parte, los entrenadores que se encuentran bajo un modelo comprensivo planteado por Bunker y Thorpe (1982) y adaptado por Kirk y McPhail (2002). En este modelo, se aboga por una metodología constructivista del aprendizaje (se parte desde el nivel que tiene el jugador), la forma jugada es la principal actividad, sin olvidarse en ningún momento de los elementos formales básicos del deporte (atacante, defensor, balón, campo de juego, etc.), incorporándolo en situaciones en las que la toma de decisiones del jugador toma mayor importancia (Cañadas, Ibáñez, García \& Sáez, 2010; Cañadas, Ibáñez, Feu, García, \& Parejo, 2011). Además, el uso de esta metodología aporta a los deportistas mayor variedad de experiencias cognitivas y motrices provocando un mayor bagaje motriz (Arias-Estero, 2008) y un menor índice lesivo (Rugg, Kadoor, Feeley \& Pandya, 2018).

La elección de una metodología repercute indirectamente en el diseño de tareas, sesiones y por consiguiente en el proceso formativo del deportista. En función de la metodología empleada, la carga total del entrenamiento será transferible a un partido (Sánchez, 2007). La carga de entrenamiento es definida como la suma de estímulos a los que se enfrenta el deportista durante un periodo concreto de tiempo (Coque, 2008). Esta carga a la que son sometidos los deportistas, en muchas ocasiones, carece de control, seguimiento o valoración de la adaptación y asimilación en el organismo. En la mayoría de ocasiones se debe al alto coste económico de los métodos tradicionales para la valoración del esfuerzo (Del Campo, Álvarez \& Lorenzo, 2008).

El control puede realizarse de diferente modo dependiendo del instrumental que se disponga. A través del análisis de la carga interna (Liberal \& García-Mas, 2011), a través de variables cinemáticas (Barreira, Robinson, Drust, Nedergaard, Raja Azidin, \& Vanrenterghem, 2016) o a través de instrumentos subjetivos como es el caso del Sistema Integral para el Análisis de las Tareas de Entrenamiento (SIATE) diseñado por Ibáñez, Feu y Cañadas (2016).

Hasta donde se conoce, no existen trabajos que analicen la fase de juego como centro del análisis de la tarea y la relación con el entrenamiento (Variables Pedagógicas y Variables Fisiológicas). Por ello, el objetivo del trabajo es analizar la relación de la fase de juego con las variables pedagógicas y carga externa en las tareas de entrenamiento en baloncesto formativo.

\section{Material y Método}

Para Montero y León (2007), la investigación se encuentra bajo un estudio empírico con una metodología cuantitativa, descriptivo mediante un código observacional diseñado con anterioridad al comienzo de dicha investigación, natural, pues estudia una variable independiente, la cual no puede ser intervenida por el investigador. Además, es un estudio retrospectivo pues únicamente se analiza la relación entre las variables.

\section{Población y muestra}

La población analizada es un equipo de Categoría Cadete que compite en categoría autonómica y cuyo entrenador tiene una experiencia superior a los 30 ańos como entrenador. La muestra está formada por 43 sesiones que conforman un total de 388 unidades estadísticas de medida (tareas).

\section{Instrumentos y material}

El instrumento utilizado para la realización del análisis de los diferentes entrenamientos fue el SIATE (Ibáñez, Feu, \& Cañadas, 2016). El tiempo se cuantificó con un cronometro marca Polar modelo M400 (Polar, Finlandia).

\section{Variables del Estudio}

La variable independiente es la Fase de Juego y se quiere conocer como interaccionan con el resto de variables en el proceso de entrenamiento. A su vez, dentro de estas variables podemos agruparlas en dos grandes grupos: Pedagógicas y Carga Externa. Cada variable está definida por un núcleo categorial y rango de apertura (Anguera, \& Hernández Mendo, 2013). Las variables del estudio están definidas en el SIATE (Ibáñez, Feu, \& Cañadas, (2016).

Se analizaron las siguientes Variables Pedagógicas (Ibáñez, 2008): i) Situación de juego de la tarea: Agrupación de jugadores que los entrenadores diseñan para cada una de las tareas; ii) Tipo de contenido de la tarea: Agrupación de los contenidos en individuales, grupales y de equipo, tanto para el desarrollo de los contenidos de la fase de ataque y defensa, así como para las conductas tácticas y gestos técnicos; iii) Medio para la iniciación al Entrenamiento: Actividades motrices deportivas que sirven para el desarrollo de unos contenidos técnicos y tácticos, cuyo objetivo es conseguir la iniciación en una modalidad deportiva; iv) Nivel de Oposición de la tarea: En el diseño de la tarea de entrenamiento. 
Tabla 1. Variables pedagógicas del estudio con su rango de apertura.

\begin{tabular}{|c|c|c|c|c|}
\hline \\
\hline Situación de Juego & Fase de Juego & Tipo de Contenido & Medio de Juego & $\begin{array}{l}\text { Nivel de } \\
\text { Oposición }\end{array}$ \\
\hline de 1 contra 0 & Ataque & $\begin{array}{l}\text { Conducta Téc-Tác Individual de Ataque } \\
\text { (CTTIA) }\end{array}$ & Ejercicio de Aplicación Simple & Sin Oposición \\
\hline \multirow[t]{11}{*}{ a $\mathrm{n}$ contra $\mathrm{n}$} & Defensa & $\begin{array}{l}\text { Conducta Téc-Tác Individual de Defensa } \\
\text { (CTTID) }\end{array}$ & Ejercicio de Aplicación Compleja & $\begin{array}{l}\text { Con Obstáculo } \\
\text { Estático }\end{array}$ \\
\hline & Mixto & $\begin{array}{l}\text { Conducta Téc-Tác Grupal de Ataque } \\
\text { (CTTGA) }\end{array}$ & Juego Simple Inespecífico & $\begin{array}{l}\text { Con Obstáculo } \\
\text { Dinámica }\end{array}$ \\
\hline & \multirow[t]{9}{*}{ Calentamiento } & $\begin{array}{l}\text { Conducta Téc-Tác Grupal de Defensa } \\
\text { (CTTGD) }\end{array}$ & Juego Simple Específico & $\begin{array}{l}\text { Con Oposición } \\
\text { Modulada }\end{array}$ \\
\hline & & $\begin{array}{l}\text { Conducta Téc-Tác Colectiva de Ataque } \\
\text { (CTTCA) }\end{array}$ & Juego Complejo Inespecífico & Con Oposición \\
\hline & & $\begin{array}{l}\text { Conducta Téc-Tác Colectiva de Defensa } \\
\text { (CTTCD) }\end{array}$ & Juego Complejo Específico & \\
\hline & & $\begin{array}{l}\text { Gesto Téc-Tác Individual de Ataque } \\
\text { (GTTIA) }\end{array}$ & Predeporte & \\
\hline & & $\begin{array}{l}\text { Gesto Téc-Tác Individual de Defensa } \\
\text { (GTTID) }\end{array}$ & Deporte & \\
\hline & & Gesto Téc-Tác Grupal de Ataque (GTTGA) I & Práctica Mental & \\
\hline & & \multicolumn{3}{|l|}{ Gesto Téc-Tác Grupal de Defensa (GTTGD) } \\
\hline & & \multicolumn{3}{|l|}{ Gesto Téc-Tác Colectivo de Ataque (GTTCA) } \\
\hline & & \multicolumn{3}{|l|}{ Gesto Téc-Tác Colectivo de Defensa (GTTCD) } \\
\hline
\end{tabular}

Téc-Tác: Técnico-Táctico

Para las Variables de la Carga Externa, se utiliza en el instrumento del SIATE (Ibáńez, Feu, \& Cańadas, 2016). Son las siguientes: i) Grado de Oposición de la tarea: En base del . número de oponentes en la tarea; ii) Densidad de la tarea: Indica la intensidad con la que se desarrolla la tarea, pudiéndose medir de forma objetiva a partir del \% de frecuencia cardíaca media de los deportistas; iii) Número de ejecutantes simultáneos: Indica el nivel de participación de los deportistas durante la tarea;; iv) Carga Competitiva de la tarea: Hace referencia a la carga emotiva, psicológica, que soporta un deportista cuando realiza una actividad con la presión por la consecución de un resultado. Cuando esta presión aparece en la tarea, éstas se convierten en actividades con una carga superior; v) Espacio de Juego: Es el lugar en el que los deportistas tienen que realizar las tareas propuestas. Su amplitud determinará la carga de la tarea al demandar que el deportista se desplace por un mayor o menor espacio. Estos desplazamientos implican una mayor activación del deportista y por tanto una mayor carga en la tarea; vi) Implicación Cognitiva de la tarea: Hace referencia a la carga táctica. La atención que el deportista tiene que tener con compańeros y adversarios, debido a las acciones de juego que realiza, debiendo prestar una mayor concentración y exigencia en la tarea; vii) Carga Total de la Tarea: Variable cuantitativa secundaria que se obtiene sumando el valor asignado dentro de cada una de las seis variables anteriores ( 1 a 5 puntos) siendo el rango entre 6 y 30 puntos. La agrupación que se realiza es la siguiente: Carga Muy Baja (6-12 puntos), Carga Baja (13-18 puntos), Carga Media (19-24 puntos), Carga Alta (25-30). 
Tabla 2. Variables de carga externa del estudio con su rango de apertura.

\begin{tabular}{|c|c|c|c|c|c|}
\hline Grado de Oposición & Densidad de la Tarea & $\begin{array}{l}\text { Porcentaje de } \\
\text { Ejecutantes } \\
\text { Simultáneos }\end{array}$ & Carga Competitiva & $\begin{array}{l}\text { Espacio de } \\
\text { Juego }\end{array}$ & Implicación Cognitiva \\
\hline Trabajo sin Oposición & Ritmo Suave o Trote & $0-20 \%$ & Actividad sin Competición & Tiro Libre & Actividades sin Relación \\
\hline $\begin{array}{l}\text { Trabajo de Superioridad } \\
\text { de } 3 \text { o más deportistas }\end{array}$ & $\begin{array}{l}\text { Ritmo Suave y } \\
\text { Continuo }\end{array}$ & $21-40 \%$ & $\begin{array}{l}\text { Concurso de Gestos de } \\
\text { Técnica }\end{array}$ & 1/4 de Campo & $\begin{array}{l}\text { Con Relación de } 1 \\
\text { comp/adv. }\end{array}$ \\
\hline $\begin{array}{l}\text { Trabajo de Superioridad } \\
\text { de } 2 \text { deportistas }\end{array}$ & $\begin{array}{l}\text { Intensidad con } \\
\text { Descanso }\end{array}$ & $41-60 \%$ & $\begin{array}{l}\text { Actividad con Oposición sin } \\
\text { contabilizar }\end{array}$ & 1/2 de Campo & $\begin{array}{l}\text { Con Relación de } 2 \\
\text { comp/adv. }\end{array}$ \\
\hline $\begin{array}{l}\text { Trabajo de Superioridad } \\
\text { de } 1 \text { deportista }\end{array}$ & $\begin{array}{l}\text { Intensidad sin } \\
\text { Descanso }\end{array}$ & $61-80 \%$ & $\begin{array}{l}\text { Act. Reducidas con } \\
\text { Oposición contabilizando el } \\
\text { resultado }\end{array}$ & Campo & $\begin{array}{l}\text { Con Relación de } 3 \\
\text { comp/adv. }\end{array}$ \\
\hline $\begin{array}{l}\text { Situaciones de Igualdad } \\
\text { numérica }\end{array}$ & Alta Intensidad & $81-100 \%$ & $\begin{array}{l}\text { Partido con todas sus } \\
\text { Variantes }\end{array}$ & $\begin{array}{l}\text { Campo de } \\
\text { Ida y Vuelta }\end{array}$ & $\begin{array}{l}\text { Con Relación de todo el } \\
\text { equipo }\end{array}$ \\
\hline
\end{tabular}

\section{Análisis de datos}

En primer lugar, se realizó un análisis descriptivo (frecuencia y porcentaje) con el fin de caracterizar la muestra. A continuación, se realizó un análisis inferencial formado por las pruebas Chi cuadrado puesto que se pretende conocer las diferencias entre las variables de tipo categórico y $V$ de Cramer para conocer el grado de asociación entre las variables analizadas. El valor de significación fue establecido en $<.05$ (Pardo \& Ruiz, 2002).

\section{Resultados}

En la tabla 3 se observan los resultados descriptivos de las variables pedagógicas agrupados por variable. Las agrupaciones más utilizadas por el entrenador son las situaciones de $1 \times 0,1 \times 1$ y $5 \times 5$. Destacan las tareas diseñadas para la fase de Ataque. En cuanto al Tipo de Contenido, este entrenador busca en las tareas Conductas Técnico-Tácticas. En cuanto al medio, aborda las tareas prioritariamente a través de Juegos. Y la mayoría de tareas se realizan con oposición.

Tabla 3. Resultados descriptivos de las variables Pedagógicas.

\begin{tabular}{|c|c|c|c|}
\hline & & $n$ & $\%$ \\
\hline \multirow[t]{13}{*}{ Situación de Juego } & $1 \mathrm{x} 0$ & 96 & 24,7 \\
\hline & $1 \times 1$ & 88 & 22,7 \\
\hline & $1 \times 2$ & 7 & 1,8 \\
\hline & $2 \times 0$ & 15 & 3,9 \\
\hline & $2 \times 1$ & 34 & 8,8 \\
\hline & $2 \times 2$ & 17 & 4,4 \\
\hline & $2 \times 3$ & 2 & 0,5 \\
\hline & $3 \times 2$ & 26 & 6,7 \\
\hline & $3 \times 3$ & 23 & 5,9 \\
\hline & $4 \times 3$ & 1 & 0,3 \\
\hline & $4 \times 4$ & 9 & 2,3 \\
\hline & $5 \times 0$ & 3 & 0,8 \\
\hline & $5 \times 5$ & 67 & 17,3 \\
\hline \multirow[t]{4}{*}{ Fase de Juego } & Ataque & 260 & 67 \\
\hline & Defensa & 81 & 20,9 \\
\hline & Mixto & 4 & 1 \\
\hline & Calentamiento & 43 & 11,1 \\
\hline \multirow[t]{3}{*}{ Tipo de Contenido } & CTTIA & 44 & 11,3 \\
\hline & CTTID & 30 & 7,7 \\
\hline & GTTIA & 51 & 13,1 \\
\hline
\end{tabular}




\begin{tabular}{|c|c|c|c|}
\hline & & $n$ & $\%$ \\
\hline & GTTID & 3 & 0,8 \\
\hline & CTTGA & 60 & 15,5 \\
\hline & CTTGD & 26 & 6,7 \\
\hline & GTTGA & 1 & 0,3 \\
\hline & CTTCA & 67 & 17,3 \\
\hline & CTTCD & 21 & 5,4 \\
\hline & GTTCA & 3 & 0,8 \\
\hline & Calentamiento & 36 & 9,3 \\
\hline & Estiramientos & 41 & 10,6 \\
\hline & Preparación Física & 5 & 1,3 \\
\hline \multirow[t]{9}{*}{ Medio } & EAS & 21 & 5,4 \\
\hline & EAC & 3 & 0,8 \\
\hline & JSI & 48 & 12,4 \\
\hline & JSE & 198 & 51 \\
\hline & JCI & 1 & 0,3 \\
\hline & JCE & 35 & 9 \\
\hline & Predeporte & 11 & 2,8 \\
\hline & Deporte & 67 & 17,3 \\
\hline & Competición & 4 & 1 \\
\hline \multirow[t]{4}{*}{ Nivel de Oposición } & Sin Oposición & 111 & 28,6 \\
\hline & con Obstáculo dinámico & 4 & 1 \\
\hline & Con Oposición Modulada & 6 & 1,5 \\
\hline & Con Oposición & 267 & 68,8 \\
\hline
\end{tabular}

En la tabla 4 se observan los resultados descriptivos de las variables de carga Externa del entrenamiento agrupados por cada variable.

Tabla 4. Resultados descriptivos de las variables de Carga Externa.

\begin{tabular}{|c|c|c|c|c|}
\hline \multirow{14}{*}{ 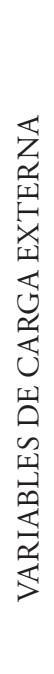 } & & & $n$ & $\%$ \\
\hline & \multirow[t]{4}{*}{ Grado de Oposición } & Sin Oposición & 122 & 31,4 \\
\hline & & Superioridad +3 & 39 & 10,1 \\
\hline & & Superioridad +1 & 66 & 17 \\
\hline & & Igualdad Numérica & 161 & 41,5 \\
\hline & \multirow[t]{5}{*}{ Densidad Tarea } & Andando Ritmo Suave & 70 & 18 \\
\hline & & Ritmo Suave y Continuo & 5 & 1,3 \\
\hline & & Intensidad con Descanso & 20 & 5,2 \\
\hline & & Intensidad sin Descanso & 226 & 58,2 \\
\hline & & Alta Intensidad sin Descanso & 67 & 17,3 \\
\hline & \multirow[t]{4}{*}{ Ejecutantes Simultáneos } & $<20 \%$ & 2 & 0,5 \\
\hline & & $41-60 \%$ & 19 & 4,9 \\
\hline & & $61-80 \%$ & 21 & 5,4 \\
\hline & & $81-100 \%$ & 346 & 89,2 \\
\hline
\end{tabular}




\begin{tabular}{|c|c|c|c|}
\hline & & $n$ & $\%$ \\
\hline \multirow[t]{5}{*}{ Carga Competitiva } & No Competición & 117 & 30,2 \\
\hline & Concurso con Gestos Técnicos & 113 & 29,1 \\
\hline & Oposición sin Contabilizar & 10 & 2,6 \\
\hline & Oposición Reducida con Resultado & 78 & 20,1 \\
\hline & Partido & 70 & 18 \\
\hline \multirow[t]{5}{*}{ Espacio Juego } & Tiro Libre & 85 & 21,9 \\
\hline & 1/4 de Campo & 55 & 14,2 \\
\hline & 1/2 de Campo & 136 & 35,1 \\
\hline & Campo & 16 & 4,1 \\
\hline & Campo + & 96 & 24,7 \\
\hline \multirow[t]{5}{*}{ Implicación Cognitiva } & Sin Relación con compañeros & 118 & 30,4 \\
\hline & Relación con 1 compañero o adversario & 127 & 32,7 \\
\hline & Relación con 2 compañeros o adversarios & 65 & 16,8 \\
\hline & Relación con 3 compańeros o adversarios & 11 & 2,8 \\
\hline & Relación con 4 compañeros o adversarios & 67 & 17,3 \\
\hline \multirow[t]{4}{*}{ Carga Total } & Muy Baja & 75 & 19,2 \\
\hline & Baja & 82 & 21,2 \\
\hline & Alta & 139 & 35,8 \\
\hline & Muy Alta & 92 & 23,7 \\
\hline
\end{tabular}

Las tareas principalmente se diseñan con un nivel de oposición de igualdad numérica, a una intensidad sin descanso, con alta participación. La carga competitiva más utilizada son tareas en las que no se compite o se realiza un concurso de gesto o conductas técnico-tácticas y suelen ser tareas diseñada en medio campo, con relación de un compañero o adversario, provocando que la carga total de la tarea sea alta.
En la tabla 5 se analiza la comparativa entre la fase de juego y el resto de variables del proceso de entrenamiento. Existen diferencias significativas en todas las variables. Las tareas con fase de ataque son diferentes a las tareas con fase de defensa en las variables analizadas.

Tabla 5. Diferencias entre la fase de juego y el resto de variables a través de Chi cuadrado y V de Cramer.

\begin{tabular}{lllll}
\hline & Chi-cuadrado & $d f$ & sig. & V cramer \\
\hline Situación de Juego & 217.878 & 36 & $.000^{*}$ & .433 \\
Tipo de Contenido & 744.844 & 36 & $.000^{*}$ & .800 \\
Medio a la Iniciación Deportiva & 445.611 & 24 & $.000^{*}$ & .619 \\
Nivel de Oposición & 146.117 & 9 & $.000^{*}$ & .354 \\
Grado de Oposición & 143.895 & 9 & $.000^{*}$ & .352 \\
Densidad & 214.630 & 12 & $.000^{*}$ & .429 \\
Ejecutantes Simultáneos & 27.897 & 9 & $.001^{*}$ & .155 \\
Carga Competitiva & 157.387 & 12 & $.000^{*}$ & .368 \\
Espacio de Juego & 200.574 & 12 & $.000^{*}$ & .415 \\
Implicación Cognitiva & 154.313 & 12 & $.000^{*}$ & .364 \\
Carga Total & 425.140 & 69 & $.000^{*}$ & .604 \\
\hline
\end{tabular}

${ }^{*} p<.05 ; \mathrm{V}>.60$ en negrita. 
El valor obtenido en la prueba $V$ de Cramer (0-1) explica el grado de asociación entre la variable con la Fase de Juego. En este apartado, destacan la asociación intensa entre las variables Tipo de Contenido (.800), Medio a la Iniciación Deportiva (.619) y la Carga Total (.604) con la Fase de Juego.

\section{Discusión}

El objetivo del presente estudio fue describir el proceso de entrenamiento y conocer si existen diferencias en las tareas en función de la fase de Juego para las que han sido diseñadas. Los principales resultados muestran que las diferencias entre ambas opciones son existentes y que diseñar una tarea para ataque o defensa repercute en el resto de variables que se tienen en cuenta. El análisis de las tareas de entrenamiento permitirá analizar el posicionamiento metodológico, así como generar teorías de entrenamiento basadas en la práctica a utilizar (Cañadas et al., 2011). De este modo, también se pretende describir cómo se desarrolla el entrenamiento deportivo desde un punto de vista técnico-táctico, conociendo los medios de entrenamiento que se utilizan, situaciones de juego o cómo son organizados los contenidos deportivos a lo largo de la temporada (Cañadas \& Ibáńez, 2010).

El entrenador a lo largo de las tareas analizadas emplea un amplio repertorio de las variables pedagógicas en las que destacan las tareas con situaciones de 1x0 (con finalidad de calentamiento, estiramientos o ejercicios de lanzamientos), $1 \times 1$ y $5 \times 5$ en cuanto a variable Situación de Juego, con finalidad de Ataque, en el que trabajan conductas técnicotácticas en cuanto al tipo de contenido, utilizando juegos como medio a la iniciación deportiva y con oposición en las tareas. En cuanto a las variables de carga externa, las tareas se caracterizan prioritariamente por ser tareas de igualdad numérica, con una intensidad sin descanso, con una participación alta, en la que o no se compite o se realizan concursos de gestos técnico-tácticos. Estas características se encuentran próximas a un perfil de entrenador con una metodología alternativa (Cañadas \& Ibánez, 2010).

En cuanto a las tareas de $1 \mathrm{x} 1$ el entrenador utiliza tareas de igualdad numérica siendo este detalle un valor próximo a métodos alternativos debido a la contextualización de tarea. En esta línea, García, Parejo, \& Cañadas (2010) propone el tratamiento igualado entre agrupaciones, comenzando tanto la tarea como la temporada con agrupaciones simples y evolucionando a medida que se avanza.

Ibáńez et al., (2016) explican que el medio de la tarea es un fiel reflejo de la metodología que se emplea. Las metodologías más próximas a modelos alternativos utilizan los juegos como medio para diseñar las tareas, mientras que los modelos tradicionales utilizan los ejercicios para diseñar sus tareas debido a que buscan una enseñanza analítica del gesto. Los juegos se caracterizan por tener un apartado cognitivo y los juegos suelen basarse en la repetición de un gesto técnico (Cañadas, Ibáńez, García, Parejo \& Feu, 2010). En este documento, el entrenador utiliza mayoritariamente Juegos Simple Específico coincidiendo con lo afirmado con Cañadas et al., (2010).

Respecto al tipo de contenido, en este documento los resultados mayoritarios se deben a tareas con finalidad de mejorar conductas técnico-tácticas coincide con la afirmación Cañadas, Ibáńez, García-Rubio, Parejo, \& Feu, (2016) que comentan que el uso de conductas provoca mejor optimización del apartado táctico y similitud con el deporte real. En contraposición, Saad y Nascimiento (2007) afirman que en la iniciación se deben de trabajar los aspectos técnicos.

Por último, la variable Nivel de Oposición diferencia también en función del modelo más próximo al que se encuentra el entrenador, Ibáńez (2008) expone que las tareas diseñadas deben de ser con oposición y con la mayor similitud al juego real. Los modelos técnicos utilizan tareas analíticas y descontextualizadas mientras que los modelos alternativos buscan enfocar las tareas con el mayor número de elementos formales del deporte (Ibáńez, 2008).

En cuanto a las variables de Carga Externa, en esta ocasión analizadas con la escala subjetiva del instrumento SIATE (Ibáńez, Feu, \& Cañadas, 2016) los resultados dependen en parte de los resultados de las variables pedagógicas. En contraposición a esta idea, Halouani, Chtourou, Gabbett, Chaouachi \& Chamari (2014) afirma que algunas modificaciones pertenecientes a variables de Carga Externa se deben a adaptaciones fisiológicas, ya que demostraron que el $3 \times 3$ en fútbol resultó de mayor intensidad que la opción $5 \times 5$. En esta línea, varios autores han demostrado que las Situaciones de Juego reducidas con un menor número de jugadores y espacio provocan una mayor Frecuencia Cardíaca que las Situaciones de Juego con mayor número de componentes (Hill-Haas, Coutts, Rowsell, \& Dawson, 2009). Por el contrario, y a favor del instrumento utilizado, Reina, García-Rubio, García-Santos, Mancha, \& Ibáńez (2018) confirman que existe relación entre la cuantificación de la carga analizada a través de instrumento subjetivo (SIATE), carga interna (Frecuencia Cardíaca) y carga Externa (Player Load). Por lo tanto, los resultados obtenidos en este trabajo son de gran validez y fiabilidad sin necesidad de altos costes económicos. En cuanto a los resultados de este documento, se otorga mayor carga total a tareas con mayor agrupación de jugadores en la situación de juego y que utilizan mayor espacio de juego. Estas afirmaciones se oponen a las anteriormente citadas. Por todo ello, los resultados obtenidos en el análisis de las tareas describen que las tareas se caracterizan por ser de igualdad numérica, de intensidad sin descanso con alta participación de los deportistas y que no se realiza competición en las tareas. 


\section{Limitaciones}

Diferentes limitaciones deben tenerse en cuenta a la hora de interpretar los resultaos obtenidos en esta investigación. En primer lugar, aunque las tareas analizadas hayan sido diseñadas por un entrenador con unas determinadas características metodológicas (tipo comprensivo), el tamaño muestral analizado es alto $(n=388)$. Además, es importante comprender que las tareas diseñadas han sido planificadas para un equipo de categoría Cadete compuesto por 12 jugadores que compiten en competición regional. Por tanto los resultados obtenidos son específicos de esta población no pudiendo ser extrapolables a otra población.

\section{Conclusiones y aplicaciones prácticas}

El análisis en función de la fase de juego muestra diferencias en el diseño de las tareas. En este caso, este entrenador, cuando diseńa una tarea con una finalidad de Ataque provoca indirectamente que el resto de variables sean diferentes que si la tarea diseñada es para la fase de Defensa. Estos resultados permiten identificar los aspectos relacionados con el proceso de entrenamiento en baloncesto formativo. La información obtenida pone de manifiesto la importancia de monitorizar y analizar las tareas y entrenamientos de los entrenadores en categorías de formación, con el fin de mejorar el proceso formativo de los jugadores. El instrumento utilizado en esta investigación (SIATE) es de acceso libre y sencillo de uso que aporta mucha información sobre el proceso de entrenamiento que se lleva a cabo pudiendo utilizarse como feedback para el entrenador.

Utilizar de manera general el uso de este instrumento en el entrenamiento podría otorgar una serie de información sobre los entrenadores pudiendo sacar conclusiones sobre conductas, metodologías procesos de entrenamientos en función de la categoría, nivel o género. Las prospectivas de futuro se centran en aumentar la muestra objeto de estudio pues existen pocos trabajos que utilicen este instrumento y que además realicen el análisis en función de la Fase de Juego, pudiendo ampliarse a otras variables. Además, sería interesante realizar análisis comparativos entre diferentes perfiles de entrenador, categorías y género entre otras variables contextuales.

\section{Agradecimientos}

Trabajo desarrollado dentro del Grupo de Optimización del Entrenamiento y Rendimiento Deportivo (G.O.E.R.D.) de la Facultad de Ciencias del Deporte de la Universidad de Extremadura.
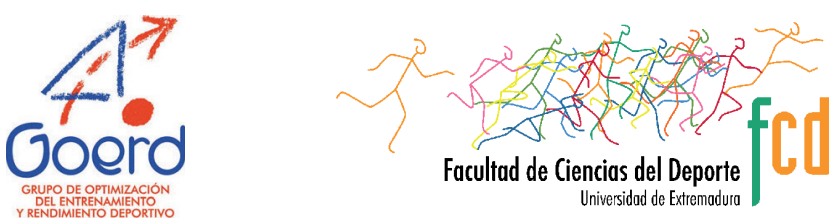

Este trabajo ha sido parcialmente subvencionado por la Ayuda a los Grupos de Investigación (GR15122) del Gobierno de Extremadura (Consejería de Empleo, Empresa e Innovación); con la aportación de la Unión Europea a través de los Fondos Europeos de Desarrollo Regional.

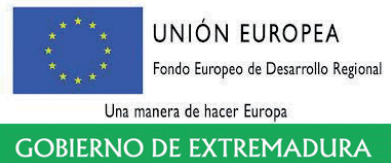

\section{Referencias bibliográficas}

1. Anguera, M. T., \& Hernández Mendo, A. (2013). La metodología observacional en el ámbito del deporte. E-balonmano. com: Revista de Ciencias del Deporte, 9(3).

2. Arias-Estero, J. L. (2008). El proceso de formación deportiva en la iniciación a los deportes colectivos fundamentado en las características del deportista experto. Retos. Nuevas tendencias en Educación Física, Deporte y Recreación, (13), 28-32.

3. Barreira, P., Robinson, M. A., Drust, B., Nedergaard, N., Raja Azidin, R. M. F., \& Vanrenterghem, J. (2016). Mechanical Player Load ${ }^{\mathrm{TM}}$ using trunk-mounted accelerometry in football: Is it a reliable, task-and player-specific observation? Journal of Sports Sciences, 1-8.

4. Bunker, D. J. \& Thorpe, R. D. (1982). A model for the teaching of games in secondary schools. Bulletin of Physical Education, 1(1), 5-8.

5. Cańadas, M., \& Ibáñez, S.J. (2010). La planificación de los contenidos de entrenamiento de baloncesto con equipos de iniciación. E-Balonmano: Revista de Ciencias del Deporte, 6(1), 49-65.

6. Cañadas, M., Ibáñez, S. J., García, J., Parejo, I. \& Feu, S. (2010) Importancia de la planificación en el entrenamiento deportivo: análisis del proceso de entrenamiento en minibasket. Wanceulen E.F Digital (7). 7. Cañadas, M., Ibáñez, S.J., García, J., \& Sáez, J. (2010). Influencia de un programa formativo en el empleo de los medios de entrenamiento en equipos de baloncesto en período de formación. Cultura, Ciencia y Deporte, 13(5), 95.

8. Cańadas, M., Ibáńez, S.J., Feu, S., García, J., \& Parejo, I. (2011) Análisis de los medios de entrenamiento en un equipo minibasket y la influencia de un programa formativo para el entrenador. Un estudio de caso. ÁGORA para la Educación Física y el Deporte, 13(3), 363-382.

9. Cañadas, M., Ibáńez, S.J., García, J., Parejo, I., \& Feu, S. (2012). Estudio de las fases de juego a través del análisis del entrenamiento deportivo en categoría minibasket. Cuadernos de Psicología del Deporte, 12(2), 73-82.

10. Cañadas, M., Ibáńez, S. J., García-Rubio, J., Parejo, I., \& Feu, S. (2016). Las situaciones de juego en el entrenamiento de baloncesto en categorías base/Game situations in youth basketball practices. Revista Internacional de Medicina y Ciencias de la Actividad Fisica y del Deporte, (49).

11. Cañadas, M., Gómez-Ruano, M.A., García-Rubio, J., \& Ibáñez, S.J. 
(2018). Analysis of training plans in basketball: gender and formation stage differences. Journal of Human Kinetics, 1-12.

12. Cassidy, T., Jones, R.L., \& Potrac, P. (2004). Understanding sports coaching: The social, cultural and pedagogical foundations of coaching practice. London: Routledge.

13. Clemente, F. M., Martins, F. M. L., \& Mendes, R. S. (2015). How coaches use their knowledge to develop small-side soccer games: a case study. South African Journal for Research in Sport Physical Education and Recreation, 37(1), 1-11.

14. Coque, I. (2008). Valoración subjetiva de la carga del entrenamiento técnico-táctico. Una aplicación práctica (I). Clinic, (81), 39-43.

15. Del Campo, J.; Álvarez, J., \& Lorenzo, A. (2008). La percepción del esfuerzo: concepto, características y aplicación al control del entrenamiento en baloncesto. En: Terrados, N., y Calleja, J. (eds.). Fisiologia, Entrenamiento y Medicina del Baloncesto. 1a edición. (pp. 121134) Badalona: Paidotribo.

16. Feu, S., \& Ibáńez, S.J. (2001). La visión del entrenador en la transición a través de deporte 2000. Revista Internacional de Medicina y Ciencias de la Actividad Física y el Deporte, 1(3), 177-187.

17. Ford, P. R.; Yates, I., \& Williams, A. M. (2010). An analysis of practice activities and instructional behaviours used by youth soccer coaches during practice: exploring the link between science and application. Journal of Sports Sciences, 28(5), 483-495.

18. García-Rubio, J., Parejo, I \& Cañadas, M. (2010). Valoración de la carga de entrenamiento. Una experiencia real con un equipo de baloncesto de Liga EBA. Revista Internacional de Deportes Colectivos (5), 4-17.

19. Giménez, F. J. \& Sáenz-López, P. (2000). Aspectos Teóricos y Prácticos de la Iniciación al Baloncesto. Diputación Provincial de Huelva.

20. Halouani, J, Chtourou, H, Gabbett, T, Chaouachi, A, \& Chamari, K. (2014). Small-sided games in team sports training: A brief review. The Journal of Strength \& Conditioning Research, 28(12): 3594-3618.

21. Hill-Haas, S., Coutts, A., Rowsell, G., \& Dawson, B. (2009). Generic versus small-sided game training in soccer. International Journal of Sports Medicine, 30(3), 636-642.
22. Ibáńez, S. J. (2008). La planificación y el control del entrenamiento técnico-táctico en baloncesto. En N. Terrados \& J. Calleja (Coord.), Fisiología, entrenamiento y medicina del baloncesto (pp. 299- 313). Barcelona: Editorial Paidotribo.

23. Ibáńez, S. J., Feu, S., \& Cañadas, M. (2016). Sistema integral para el análisis de las tareas de entrenamiento, SIATE, en deportes de invasión. E- balonmano. com: Revista de Ciencias del Deporte, 12(1), 3-30.

24. Kirk, D., \& McPhail, A. (2002). Teaching games for understanding and situated learning: Rethinking the Bunker-Thorpe model. Journal of Physics Teacher Education, (21), 177- 192.

25. Liberal, R. \& García-Mas, A. (2011). Percepción de dolor y fatiga en relación con el estado de ánimo. Cuadernos de Psicología del Deporte, 11(2), 93-117.

26. Montero, I. \& León, O.G. (2007). Una guía para nombrar los estudios de investigación en psicología. Revista Internacional de Psicología Clínica $y$ de la Salud, 7 (3), 847-862.

27. Narazaki, K., Berg, K., Stergiou, N., \& Chen, B. (2009). Physiological demands of competitive basketball. Scandinavian Journal of Medicine \& Science in Sports, 19(3), 425-432.

28. Pardo, A., \& Ruiz, M. A. (2002). SPSS 11. Guía para el análisis de datos. Madrid: Mc Graw Hill.

29. Reina, M., García-Rubio, J., García-Santos, D., Mancha, D., \& Ibáńez, S.J. (2017). Análisis correlacional de la carga objetiva y subjetiva de entrenamiento en baloncesto femenino. Presentado en el $13^{\circ}$ Congreso Internacional de Ciencias del Deporte y la Salud (Pontevedra).

30. Rugg, C., Kadoor, A., Feeley, B. T., \& Pandya, N. K. (2017). The Effects of Playing Multiple High School Sports on National Basketball Association Players' Propensity for Injury and Athletic Performance. The American Journal of Sports Medicine, 0363546517738736.

31. Saad, M. A., \& Nascimento, J. V. (2007). Estruturação das sessões de treinamento técnico-tático nos escalóes de formaçáo do futsal. Revista Portuguesa de Ciências do Desporto, 7(Supl. 1), 21-8

32. Sánchez, M. (2007). El acondicionamiento físico en baloncesto. Apunts, Medicina de l'Esport, 42, 99-107. 